\title{
A CONTRIBUTION TO THE SURGERY OF THE BRAIN.
}

\author{
By WILLIAM THORNLEY STOKER, Surgeon; and GUY \\ L'ESTRANGE NUGENT and JOSEPH O'CARROLL, \\ Physicians ; \\ Richmond, Whitworth, and Hardwicke Hospitals.
}

[Read in the Section of Surgery, May 9, 1890.]

The two cases about to be recorded are ones respectively of tumour and of abscess in the cerebrum. Although operation in the former yielded but temporary benefit, yet it is of scarcely less interest than the latter, in which operation was attended by complete success. Both have a bearing on the diagnosis, localisation and treatment of the classes of complaints to which they belong. Our failures in surgery are generally more instructive than our successes, for calamity is a more direct guide to danger than is success. One is positive, the other negative, in its value. The limited measure of success, therefore, which has attended one of these cases offers the stronger reason why they should both be recorded.

The first case is one of tumour in the superior parietal lobule on the right side, which was diagnosed and operated on without success, the relief being but temporary, and the patient dying three weeks after operation.

The second case is one of abscess in the right temporal lobe, which was diagnosed and operated on, and the subject of which is now alive and doing well.

CASE I.-J. B., aged forty-two, a boiler riveter, was admitted into the Richmond Hospital on December 12th, 1888. He was of muscular, vigorous habit, temperate, had not had syphilis, and, until the day of his admission, suffered from no blemish except slight deafness, produced by his noisy occupation. He was a 
widower with two children, and a man of quiet, regular habits. His father died of phthisis, his mother, probably, of malignant disease of the stomach.

On December 12 th he left work at 5 o'clock, p.m., feeling perfectly well. When walking home the lace of his boot became loose; he stooped to fasten it, and when raising himself became giddy. $\mathrm{He}$ recovered himself in a few minutes, but his left leg began to jerk, the movements commencing at the instep and gradually extending up the extremity, and engaging the same side of the trunk. He was unable to walk, had to catch the railings to prevent himself from falling, and feared that the passers-by would think he was drunk. He was assisted home, and brought to hospital the same evening.

When admitted he was, and had been, perfectly conscious and intelligent. He was sweating profusely; his pupils were dilated and symmetrical. His left leg was violently jerked by clonic spasms, occurring from 30 to 40 times in the minute, and engaging the extensor muscles on the front of the leg and thigh, and the adductors of the thigh. The limb was extended and rotated outwards. The left side of his trunk was engaged in the spasms to such an extent that he described them as "taking his breath away." The muscles about the left shoulder were affected, but to a much slighter degree. His face was turned to the right side. There was complete loss of sensation over the affected parts on the left side. He complained of much pain during the spasms. The kneejerk was exaggerated on the left side.

His bowels were emptied by an enema, and he was put on large doses of bromide of potassium. After a few hours he was greatly relieved, and the spasms confined themselves to the leg.

December 13th.- -Spasms are confined to the muscles on the front of the leg; sensation is completely recovered except on the front of the leg, where it remains slightly impaired. He is distressed by pains in the front of the leg and dorsum of foot; face slightly contracted on the right side.

From the time of his admission on December 12 th his condition was one of steady improvement. His treatment consisted mainly in frictions to the left lower extremity, with a diluted ointment of atropia to relieve his severe pain; in the use of bromide of potassium, and attention to his bowels. His pain lessened; the spasms gradually disappeared in order from above downwards-the shoulder being the first, and lower extremities the last, to recover. The 
pain and spasm remained in the leg after the thigh had recovered, then in the dorsum of the foot, and lastly in the great toe. He had no ocular symptoms, no headache, but his speech was, probably, a little more slow than it should be.

On December 16th he was able to leave his bed, but could not walk; on December 20th he was able to walk, and on January 8th he insisted on leaving the hospital and resuming his work, although we were anxious to keep him under observation.

The opinion formed of his case at the time was that he had probably a tumour near, or in, the leg centre in the right hemisphere; but the absence of ocular symptoms, of vomiting, and of headache rendered the diagnosis uncertain.

$\mathrm{He}$ remained well and at work until April 21 st, 1889, when, as he was standing in the street, he was seized by violent clonic spasms of the muscles of the entire of the left side, except thiose of the face. The attack lasted for an hour, and then passed away. Two hours afterwards he got a second attack, even more violeut than the first, and was brought to hospital. His condition was almost similar to that when under observation the previous December, but more pronounced. The differences were that his head was drawn to the left side, and he had severe frontal headache. He did not sleep for two days after his admission, and then only as a result of the use of bromide of potassium.

On April 30th, seven days after his admission, he was able to get up, the spasms having diminished in frequency and in degree, and being at this date only present a few times in the day, and confined to the leg. His temperature, when admitted, on April 21 st, was $101^{\circ}$; it remained without much rise or fall, between $99^{\circ}$ and $100^{\circ}$, until the $28 \mathrm{th}$, when it became normal, and remained so. He was removed to the Whitworth Hospital, under the care of Dr. Nugent, on May 6th, his condition being then as follows :-

His left lower extremity was weak, but he could stand and move with the aid of a chair. He could not move the toes of the left foot. He had numbness of the leg; the plantar reflex was present; the knee-jerk of the left leg was exaggerated. He could flex the leg, but could not retain it in the flexed position. He suffered from giddiness, which disappeared when he had been out of bed for an hour or so.

This state of affairs lasted with little ups and downs, his condition on the whole being an improving one, until May 20th, the 
only marked inconvenience he suffered being from a severe pain on the inside of the thigh. On May 20th he got another attack of spasm. The movements commenced in the knee and worked up the left side to the head. He complained of great pain over the stomach, and of severe frontal headache; had twitchings of the extensor muscles of the thigh, of the abdominal muscles, the pectorals, and trapezius of left side. No twitchings of arm, face, or tongue.

May 23rd.-Better ; twitchings have ceased in trapezius and pectorals; muscles still affected are adductor longus, tensor vaginæ femoris, rectus abdominis, external intercostals-all of left side. Could walk across the ward without assistance; left side weak ; he brings muscles of right side into play, and swings left limb in the arc of a circle.

May 24th.-Is much better; movements cease during sleep; had slight clonic spasm of the adductor longus, rectus abdominis, external oblique, external intercostals, and scalenus anticus of left side. The spasms occur about twenty times in the minute; they disappeared in order from above downward, the adductor longus being the last to recover.

From May 24th to June 13th he continued in much the same state-able to be up for the greater part of the day, sleeping well at night, suffering from numbness on the front and outside of leg, occasional giddiness and spasms at times, of a mild character, and of short duration. The spasms always commenced in the foot, extended to the thigh, came on suddenly, were attended by pain and perspiration, lasted from five to ten minutes, and were followed by weakness and numbness in the parts.

June 13th.-Had an attack of spasm in the left leg, which lasted, at intervals, for two hours.

June 20th.-Had an attack of clonic spasm, affecting only the left arm, deltoid, triceps, and trapezius muscles, which lasted off and on until July 2nd. Through July he remained in much the same state, and, on August 1st, had a severe attack of spasm. His arm was jerked at a rapid rate from the elbow up, his head turned to the left side, his eyes open, and he could not speat for several minutes. The attack lasted for over an hour, and was followed by loss of power in the hand and arm, and by severe and constant pain in the shoulder.

August 8th.-Spasms, chiefly engaging the trapezius, deltoid, biceps, and supinator longus. 
August 10th.-The loss of power in the hand persisted; all the muscles of the upper extremity responded to electrical stimulus.

August 15th.-The left side of the face was paralysed.

August 19th.-Intellectual powers are failing; the patient does not know what he is speaking about; he cannot return an intelligent answer. His tongue is normal, and protruded straight. He has, for the first time, complete loss of sensation in the arm.

From August 19th his condition was one of rapid deterioration. His patellar reflex was still exaggerated on the 21st. His eyes were examined by Mr. Swanzy on the 23rd, who found no optic neuritis, or evidence of disease. On the 28th he passed urine unconsciously, but had some power of giving notice when the bowels were about to act. He was in a state of semi-consciousness, lying quite helpless, and dead to his surroundings, unless roused. When roused, and asked if he had pain, he put his hand, slowly, to the right side of his head and gave a stupid assent. When loudly and repeatedly desired to put out his tongue, he did so slowly, but in the case of each movement did not attempt to recover himself, leaving his hand at his head in the one case, and allowing his tongue to remain protruded in the other. He swallowed slowly and with difficulty. His temperature was normal, $98.4^{\circ}$; his pulse and heart wreak.

The next morning, the 29th of August, he was so much worse that it was evident he would soon die if operation were not undertaken at once, and so we proceeded to trephine the skull and explore the brain.

Previously to operation he was unable to speak, could not swallow, was completely paralysed, both as to motion and sensation on the left side, including the face; his pupils were dilated and symmetrical; he had nystagmus; and could be roused only by great effort to make any response, his extreme of intelligence being to put his right hand to his head when loudly questioned as to the existence of pain; he retained his hand at his head until it was removed from it.

Operation.-As the leg centre was pointed to by the clinical history of the case as the starting point of the disease, its situation was selected for operation. The flap of scalp was cut in a semicircular fashion, with its convexity corresponding to where the inner edge of the trephine hole would be-the advantages of forming the flup in this fashion being, that it leaves its chief vascular and nervous supplies, which are from below, unimpaired, and that 


\section{A Contribution to the Surgery of the Brain.}

the size of the flap can be indefinitely extended by carrying the incisions downwards towards the ear in case additional apertures in the skull are required. The trephine, 1 inch in diameter, was first applied over the upper part of the fissure of Rolando, as near to its upper end as the safety of the longitudinal sinus would allow. A second trephine opening was made, antero-inferior to the first, in the line of the fissure of Rolando, and the angles of bone being removed with a forceps, an oval opening, 1 inch in diameter and 2 inches in length, was obtained. When the dura mater was incised the brain bulged in the characteristic fashion seen in cases of great intracranial pressure. No evidence of diseased structure could be seen in the exposed area of brain. The region of the leg centre was then explored both with a probe and a trocar, and as nothing evidencing disease was found the entire motor area was then similarly examined, but without result. The intracranial pressure was so pronounced that when a silver probe was introduced into the brain substance, and left there, it was rapidly extruded by the force exercised by the brain.

It became evident that no good could result from further exploration; so the membranes were replaced, the flap adjusted by points of suture, a rubber drainage-tube passed across its base, and an antiseptic compress applied over all. I did not replace the bone, because it was now evident that the operation could give even temporary relief only by allowing some degree of protrusion so as to relieve the intracranial pressure.

His temperature, which had previously been normal, rose on the erening of operation to $101^{\circ}$, and the next day, August 30th, to $103 \cdot 5^{\circ}$. It then fell so as to become normal on the third day after operation, and remained so until August 17th, when it began to rise, and by the day of his death, September $19 \mathrm{th}$, had risen to $105.4^{\circ}$ It was essentially the same on both sides all through the case.

August 30th.-Speech and intelligence improved; some tonic spasm of right side of face; requires to have a catheter passed; nystagmus continues.

August 31st.-Speech and intelligence much better; passes water easily; takes nourishment freely; complains of great pain in the head; can open and close both eyes symmetrically when the tonic spasm of right side of face is absent.

September 1st.-Drainage tube removed; general condition improved; power returning in left arm. The wound has all 
united, except the apertures where the drainage tube passed through. The surface of flap raised above surrounding skin by pressure of the brain.

September 2nd.-Tonic spasm of left side of face.

His condition from September 2nd to September 8th was one of improvement. He was fairly intelligent, had improving power over the left arm, and continued to have an oozing of softened brain substance through the holes where the drainage tube had been; the relief from pressure thus obtained being the cause of his temporary improvement. On the night of September 8 th he was restless and semi-delirous, tore off his dressings, and opened the flap, so that next morning we found a large hernia cerebri. $\mathrm{He}$ was, so far as mental condition and paralysis were concerned, in no worse condition than before this accident. From this date, however, he began steadily to disimprove. A large destruction of the protruding brain and, as post-mortem examination afterwards showed, of the neighbouring substance took place by suppuration and sloughing in spite of every antiseptic measure we could use. The condition of paralysis of the left side from which he had partially recovered, so far as his face, trunk, and upper extremity were concerned, returned; and, on September 18th, he became completely hemiplegic, passed into stupor, finally into coma, developed hiccough, cerebral vomiting, preagonistic temperature, and died on the following day, September 19 th.

Post-mortem, September 21st. - The hernia cerebri formed a protrusion about 3 inches in diameter and 1 inch beyond the level of the skull. The calvarium separated from the dura mater with normal readiness. Around the trephine opening the membranes were adherent to each other, so as to seal the cavity of the arachnoid. There was pronounced arachnitis about the base of the brainmost marked under the cerebellum, the medulla oblongata, and the anterior lobes of both sides, but more extensive on the right side, where the inflammation was not merely basic but extended up on the frontal convolutions.

Corresponding to the trephine opening at the fissure of Rolando, and in the centre of the hernial mass, was a ragged, sloughing, suppurating cavity, extending for more than an inch into the brain substance, but not engaging the ventricular cavity. On section, a tumour was found lying immediately behind this cavity, and occupying the posterior portion of the parietal lobe. It approached within half an inch of the longitudinal fissure, and within a quarter 
of an inch of the surface of the cerebrum. It was spherical in form, about one inch in diameter, and of equal consistence and resistance to normal brain matter.

The tumour has been examined by Professor Purser and Dr. Conolly Norman. It is a spindle-celled sarcoma.

In addition to the value which in the present unripe state of our knowledge of brain tumours attaches to every case which can add to clinical or statistical information, there appear to be some points of special interest in this record. As regards the clonic spasms, although intermission in their occurrence is the rule, the intermissions here were of an unusual character. They were irregularly extended over a very long period, and showed ups and downs of intensity instead of the progressive gravity which is generally seen. Besides, although they commenced in the leg and indicated the vicinity of the upper end of the fissure of Rolando as the focus of disease, and although these leg symptoms were followed by arm phenomena, and finally by engagement of the face-the natural sequence where a growth commencing in the centre for the leg gradually involved the centres for the upper extremity and face in the natural order of their anatomical relation-yet it was clinically and physiologically discrepant to find the arm or muscles of the trunk sometimes in a state of spasm, while the lower extremity, although not paralysed, was at rest. It is additionally out of rule to find that post mortem evidence discovered the tumour behind the situation where it might reasonably be expected to lie. The lessons derived from this part of the case appear to be:-

1. That the leg-centre extends further back than is usually taught into that part of the parietal lobule where its extension has been regarded as doubtful.

2. That the centre for the leg muscles is behind that for those of the thigh, as in this case the earliest muscles affected were those below the knee, and we may fairly conclude that the disease first engaged the part of the motor area next itself.

3. That what may be called referred pressure-that is to say, pressure remotely exercised-is a powerful and confusing factor in 
the examination of brain tumour. For here it is evident not only that the small localised growth on the back of the leg-centre proceeded to engage the centres for the arm and face, but, what is even more remarkable, that it was able to affect them at times when its pressure spared and seemed to skip the intermediate and more closely related centre for the lower extremity.

The next point of great interest seems to be the absence of three out of that group of four symptoms which we are in the habit of relying on generally as indicative of tumour of the brain-namely, hemi-spasm, vomiting, optic neuritis, and fixed headache. Of these only the first was present; there was no optic neuritis, no vomiting of a cerebral character, and headache was a late, an occasional, and inconstant symptom.

It was this hiatus in the clinical history that caused so long a delay in adopting operation. This delay having occurred, it is satisfactory to know that it was not the cause of the loss of the case, as the nature and situation of the tumour would have rendered operation futile, however early it had been performed. All that surgical interference did was to prolong the life of a man who was dying when operated on, and for a period to restore him to consciousness.

So far as our present knowledge goes the future of brain surgery affords no hope of cure in cases like this. To have a successful issue in such operations two conditions must exist-first, an accurate localisation of disease ; and, second, that the neoplasm should be of the nature of a cyst or encapsuled growth, or so surrounded, or so differentiated in structure from brain substance, that its position can be felt or determined by instruments or by touch when the eye cannot reach it. It is obviously almost impossible to discover a tumour which cannot be seen, and which, like this, is traversed by instruments with just the same degree of resistance as the surrounding brain; and, even if it be discovered, it is quite impossible for the operator to know whether it has been entirely removed. 


\section{$268 A$ Contribution to the Surgery of the Brain.}

CASE II.-E. W., tailoress, aged eighteen years, was admitted to the Whitworth Hospital, under the care of Dr. O'Carroll, on March 1st, 1890.

She was one of a family of eleven children, of whom seven were dead, and she had enjoyed good health up to four months previously to her admission, when she was attacked by pain in her right ear, followed by a purulent discharge from the meatus, and attended by impairment of hearing. She then began to lose appetite, to waste, to vomit, and to suffer from severe occipital headache. The vomiting increased in frequency, until it attacked her four or five times a day, the headache being relieved by the act of vomiting.

When admitted she was extremely emaciated, pallid, and presented an appearance indicative of great suffering and much exhaustion. There was a copious purulent discharge from the right ear, but no local tenderness or redness. She had from four to six attacks of vomiting daily, and had almost constant pain in the back of her head, which showed a paroxysmal character, and became at times so severe that it caused her to cry aloud in the most piteous fashion. She was seized five or six times a day by tonic spasm of the muscles of the back of her neck, causing extreme retraction of the head and attended by most distressing pain. These spasms were more frequent in the night than in the day, and lasted from five to ten minutes. During them her usual attitude was sitting up in bed, holding its rail, with her legs drawn up, and her left fingers alternately flexed and extended. At the end of the attack she generally turned over on her left side into the semiprone position, her eyes at this period showing conjugate deviation to the right. She felt as if "the bed was going from under her," and was giddy when walking. Her right pupil was at times somewhat larger than her left. She had been hypermetropic from her school days. Ophthalmoscopic examination showed double optic neuritis. The reflexes were present, but diminished by emaciation. Her temperature in the axilla averaged $97^{\circ}$. She was partially deaf on the right side, and had right anosmia, not being able to distinguish assafoetida or iodoform with the right nostril.

She remained in the Whitworth Hospital until March 8th, when she was transferred to the Richmond Hospital for surgical treatment, her condition having become extreme, and Dr. O'Carroll having made up his mind that an intracranial abscess existed.

Examination of the case will show that sufficient grounds were 
present for this opinion. The points of evidence may be summarised as follows :-

1. The previous existence of inflammation and suppuration, engaging the middle or internal ear.

2. Persistent headache.

3. Cerebral vomiting.

4. Giddiness.

5. Optic neuritis.

6. Rigidity of the muscles of the back of the neck and retraction of the head.

As to where the abscess was situated some doubt existed, as the ocalising symptoms were less definite than those of a general character. As intracranial suppuration resulting from ear disease usually occurs either in the temporal lobe, on one hand, or in or about the cerebellum, pons, or medulla, on the other, it was fair to assume that if pus were present it would probably be found in one of these situations. In favour of the pus being siturted in the cerebrum, was the anosmia, which would probably indicate some engagement of the anterior extremity of the uncinate convolution of the side on which the loss of smell was observed.

On the other hand, the relation of disease to the cerebellum, or, at all events, to the parts below the tentorium, was indicated by the marked rigidity of the neck muscles, the retraction of the head, and the frequency of the vomiting-all conditions which are observed to attain their greatest development when the pons or medulla are subject to pressure from suppuration. Again, the giddiness (cerebellar incoördination) pointed to pressure on the cerebellum. Also the absence of auditory phenomena on the left side, as affording evidence of freedom of the right superior temporal convolution, gave negative inference that the temporal lobe being free, the seat of disease was probably cerebellar.

On weighing the evidence the conclusion arrived at was that pus probably existed in the temporal lobe, and that if not found there it should be sought for below the tentorium.

March 9 th.-Operation, having been decided on, was performed under chloroform, and was planned so as to give access to the temporal lobe, and to permit of a trochar being passed down through the tentorium into the substance of the cerebellum, provided pus were not discovered in the cerebrum. A careful observation of Professor Cunningham's casts of the brain, and of Professor Fraser's photographs, having shown Mr. Thornley Stoker that the 
point selected by Mr. Barker for his trephine opening in these cases would not expose, as he supposed, the middle temporo. sphenoidal convolution, but would expose the inferior convolution so low down as to endanger the lateral sinus, Mr. Stoker took a point higher up-that is to say, a horizontal line being taken from the lower border of the anterior bony nares through the middle point of the meatus, $1 \frac{1}{4}$ inches are measured from the mid-meatus point back along this line, and from this point a perpendicular raised for $1 \frac{1}{2}$ inches. The base line here used, passing from the lower border of the anterior bony nares instead of from the lower edge of the orbit (Reid's), as used by Mr. Barker, gives an additional elevation of about $\frac{1}{4}$ inch, and if the point be taken $1 \frac{1}{2}$ inches, instead of $1 \frac{1}{4}$, above it, a total elevation of about $\frac{1}{2}$ inch above Mr. Barker's point is reached. A trephine opening made here will usually expose the middle convolution, and will give easy access to any part of the temporal lobe, while it is high enough to enable an operator who desires to explore the cerebellum to do so by passing a trochar downwards, inwards, and backwards, without risk of wounding the lateral sinus.

Taking the point thus indicated as a centre, a curved incision was made above it, a flap turned down, the periosteum resected from a circular area, and a trephine opening 1 inch in diameter made. The dura was then divided, when the brain-substance protruded in the fashion usually seen where undue intracranial pressure exists. The temporal lobe was next explored with a trochar and cannula $8 \mathrm{~mm}$. in circumference. The instrument was introduced in various directions, as far as seemed allowable without the risk of passing beyond the lobe in front, or into the ventricular cavity internally. Puncture was made nine times unsuccessfully, but on the tenth attempt pus was found two inches from the surface of the brain, in a direction downwards, inwards, and backwards from the trephine opening, at a point corresponding to the junction of the temporal and occipital lobes immediately above the tentorium. As the pus was very thick and exuded slowly, an aspirator was attached to the cannula, and about 4 drachms of pus withdrawn. The cavity was then washed through the cannula with a solution of boracic acid, and, the cannula being withdrawn, a rubber drainagetube $12 \mathrm{~mm}$. in circumference was introduced along its track by a Lister's forceps. The dura was then replaced, the flap sutured, and the end of the drainage tube left protruding through its most depending angle. A dressing of sublimated gauze covered with a 
bulky pad of sublimated wood wool was applied, and retained in position by a linen cap made to fit the skull loosely and tied under the chin by strings.

The patient got almost complete relief from pain and spasm after the operation, and had no bad symptoms until 7 o'clock next morning.

March 10th.-She became very restless, got severe occipital pain and symmetrical spasms of the muscles of the back of her neck, attended by retraction of her head. She became drowsy and semi-conscious, and experienced great difficulty in swallowing. Her temperature, which was $99 \cdot 2^{\circ}$ at the time of operation, had now risen to $100 \cdot 6^{\circ}$. The dressings were removed; considerable bulging of the flap was found to exist, and some slight oozing of blood and brain subtance to have occurred through the tube. The tube was withdrawn, a stitch removed at each angle of the wound, and a considerable amount of clotted blood and softened brain matter pressed from under the flap. A small rubber drainage-tube was passed between the openings made by the removal of sutures, and as some heat and glazing of the skin were present, wet sublimated gauze-dressings were substituted for the dry ones previously in use.

March 11th.-Temperature $104^{\circ}$; not so much drowsiness; no pain in occiput, or spasm of neck ; complained of pain in teeth and eyes, and of dryness of mouth and throat, and suffered so much difficulty in swallowing that she was chiefly fed by nutrient enemata and zyminised suppositories. When the dressings were removed a considerable oozing of brain substance and blood was found to have taken place.

March 11th to 22nd.-The relief from pressure now produced much amelioration in her condition. Her temperature gradually fell, and, until March 22nd, varied between $98^{\circ}$ and $100^{\circ}$, reaching $101^{\circ}$ on one day only. She remained in a fairly comfortable state until March 22nd, her chief inconveniences being due to pain in her teeth, dryness of the mouth and fauces, difficulty of swallowing, and a herpetic eruption at the right angle of her mouth. Pus began to be discharged on the 13th, and on the 17th the flap had become detached, and a hernia of the brain substance projected through the trephine hole. It was found impossible to do more than limit the size of this protrusion by moderate pressure, as when reduced and retained within the skull, severe pain and return of the old symptoms demanded that it should be allowed to protrude. On March 22nd urgent symptoms showed themselves, and 
from this on to the close of the case its history is a surgical romance of hairbreadth escapes of a very remarkable character.

March 22nd. - Temp., which on the previous day had been $98^{\circ}$, rose suddenly late in the evening to $104^{\circ}$. She was seized by a sudden and violent return of the old pains in the back of her head, attended by retraction as formerly. Her condition became so extreme that it was feared she was dying, and in Mr. Stoker's absence his colleagues-Messrs. Thomson, Corley, and Dr. O'Carroll-saw her in consultation, and concluding that fresh intra-cranial suppuration was the probable cause of trouble, probed the brain in the direction of the former abscess, but without result. Some pus, however, was pressed out from between the hernia and the posterior edge of the trephine opening.

March 23rd.-This morning the girl's condition not being sensibly improved, and some stupor existing, she was put under chloroform, the hernia removed on a level with the skull by curved scissors, and on careful probing a purulent cavity found to exist between the dura and bone, posterior to the opening. This had been sealed by the overlapping hernia, and the retention of pus had caused the advent of the fresh symptoms. A rubber drainage tube was placed in this cavity, the flap readjusted, and pressure applied. The effect of the drainage was immediate relief from pain, spasm, and stupor; a fall of temperature from $104^{\circ}$ to $98^{\circ}$, and of pulse from 140 to 110 .

March 23rd to April 12th.-From this date until April 12th a steady improvement took place. As some tendency to protrusion of the brain still existed, an elastic bandage was applied over the gauze dressing; it reduced the hernia in a few days, and being daily reapplied, prevented any further trouble in that direction until the aggravation of symptoms acquired its removal on April 28th, after which date the hernia reappeared. On April 5th the girl was so well that she was allowed up for a short time. On April 7 th the epidural suppuration had ceased, the cavity closed, and the dura united to the bone, so that a probe could no longer be passed between them.

April 9th.-Exposed surface of brain and bone is granulating in the most healthy way. Her general health and comfort is good, and no pus appears on the dressings except such as secreted by the surface granulations.

From April 13th she complained of pain in her forehead, eyes, teeth, back of neck, and right arm. These symptoms gradually 
increased in severity. The temperature, previously normal, rose on the $17 \mathrm{th}$, and on that and the four following days raried between $102^{\circ}$ and $103^{\circ}$. On April 21 st she suffered from vomiting and difficulty of breathing, and on April 21st the cause of these sufferings became evident, for on exploring the granulating brain with a probe a sequestrum from the edge of the trephine hole, forming about two-thirds of a circle, was easily found and withdrawn. Its removal, however, failed to give relief, for although the temperature fell, the pains and dyspnoea persisted, the breathing became slow and irregular, and spasms occasionally attacked the right arm and shoulder. She became quite intolerant of the elastic bandage round her head; it was necessary to remove it, and the hernial protrusion again took place. As localised cerebritis seemed to exist and be extending, she was on April 29th put on a mercurial course, 3 grains of grey powder and $\frac{1}{4}$ grain of opium being given three times a day. The temperature now had an average of $99^{\circ}$, sometimes falling to between $97^{\circ}$ and $98^{\circ}$, and occasionally rising to between $102^{\circ}$ and $103^{\circ}$.

May 2nd.-On removing the dressings an area as large as a sixpence of the upper and back part of the hernia was found to be gangrenous, with an aperture in the centre of the slough discharging pus. On introducing a probe it entered an abscess cavity in the brain in a direction forwards and inwards for about $1 \frac{1}{2}$ inches, which, allowing for the projection of the hernia, would mean more than an inch within the cranial wall. The cavity was washed out through an incision made in the gangrenous tissue, a drainage tube inserted, and gentle pressure applied. The breath having become mercurial, the grey powder was reduced to one dose per diem.

May 3rd.-The slough had localised itself, and measures $1 \frac{1}{2}$ in. by 1 in. The mercury was stopped.

May 4th to 6th.-Condition rapidly deteriorating; occasional cerebral vomiting; stupor passing into coma; Cheyne-Stokes' respiration; pulse 130; limbs paralysed; right pupil dilated; left contracted; conjunctival reflex gone; she appeared to be dying. At noon on May 6th a free discharge of very fœtid pus took place, followed by a rapid return to consciousness; the breathing became quiet and regular; vomiting ceased; all paralysis disappeared, and the pupils became symmetrical.

May 7th.-Discharge of pus small and healthy; general condition most favourable; hernial tendency very slight, being easily overcome by elastic pressure, which was now resumed and used without 
inconvenience until the end of the treatment. The temperature became normal on May $7 \mathrm{th}$, and remained without any remarkable change until May 13th. ${ }^{\mathrm{a}}$

May 7 th to 13 th. - Between these dates very satisfactory progress was made, the abscess cavity, into which a probe could be passed for $1 \frac{1}{2}$ inches, was daily washed out with boric solution, and several small sloughs of brain substance thus removed. A small sequestrum from the inner table of the skull was also discharged. The amount of pus gradually diminished.

On May 13th she began to complain of frontal pain. She rolled her head from side to side, the ward seemed to her to be turning round. She could see plainly, had photophobia, diplopia, pain in eyeballs, and the gas lights appeared to be of various colours. Temperature, $103^{\circ}$. On the next day, May 14th, the temperature had risen to $104 \cdot 6^{\circ}$, and the conditions just mentioned were even more pronounced.

May 15th.-A free discharge of pus took place, and proved to be the last cerebral incident in the history, for it was followed by a return to nearly normal temperature; and from this date a steady and permanent improvement took place, which has resulted in complete relief from all cerebral trouble.

The clinical history of the brain disease ends here; for, although the girl was retained in hospital until August 7th for the purpose of observation, and for the treatment of lung disease, no further head symptoms showed themselves. The wound healed completely, the flap of skin was replaced over the trephine opening by the natural cicatrisation along its edge, and the surface of the scalp over the aperture in the bone soon assumed a slightly depressed character.

In the early part of June, although no cerebral symptoms were present, a hectic temperature made its appearance, and caused much conjecture as to its cause. A cough with purulent expectoration appeared, and, on examination, a small cavity was found in the apex of the right lung. No tubercle bacillus was found in the sputum, and it was hoped that the condition might be pneumonica hope apparently justified ; for, under treatment by dry inhalation of carbolic acid and creasote, the expectoration diminished, the temperature fell, and in August she was sent to the Convalescent Home at Stillorgan, in a very fair condition of general health.

" In preparing this paper for the press the history has been completed up to the present date, Nov. 10th, 1890. 
She has since been kept under observation, and now, Norember 10th, her condition is as follows:- - Her mental condition is perfect; she has no paralysis of any form; her sight is as good as before her illness; hearing in the left ear is perfect, and in the right impaired so far as is usual where the middle and the internal ear have been the seat of suppuration. There is at present evidence of lung disease at both apices. She has lately spat blood, but the tubercle bacillus has not been found in the sputum.

As regards the suppurating ear, subsequent to operation the purulent discharge was for a long time abundant. The ear was syringed twice a day with boric sclution, and by the time convalescence had taken place the discharge had ceased. No local tenderness, redness, or other indication appeared which would have justified an operation to penetrate the mastoid bone.

Daily records of medical treatment in this case have not been given, because they would be tiresome, and not essential. Morphia and bromide of potassium were both used at various times for the relief of pain and spasm, and each seemed to have a good effect, and not to produce any evil consequence.

In addition to the value of this case for diagnostic and operative reasons, its clinical history has several matters of much interest, not only from a physiological point of view, but also as bearing on management, and as showing how far from hopeless even an apparently desperate case is. The engagement of the origins of the fifth and eighth nerves after the operation, as shown by the pain in the teeth, the dryness of the mouth, the facial herpes, and the difficulty of swallowing, speaks plainly as to the extent of brain substance engaged in the neighbourhood of the abscess. Again, the recovery of the brain from so extensive a destructive process as existed here is very remarkable. Not to speak of the amount of pus discharged, or of the numerous secondary abscess cavities which penetrated the brain in different directions, and each one of which seemed sufficient in itself to cause death, there was by sloughing alone, and by the removal of the hernia cerebri, an amount of direct destruction of brain substance which cannot have been less than an ounce, and may have been much more. And yet, with this extensive loss of material, no paralysis has been 
produced. Clearly, the temporal lobe is largely superabundant, and capable of considerable curtailment without much ill effect.

Concerning the hernia a word may be said. The effect of the elastic pressure here was so good that it teaches how slow a surgeon should be to remove such a protrusion. It is to be remembered that when irritation and inflammation have subsided there will be abundant room in the skull for the brain substance in such a case as this, where no new growth is present. Had elastic pressure been used from the time of operation it is extremely unlikely that the outgrowth would have taken place. But whether this be so or not, it should be remembered that a hernia cerebri, although perhaps rebellious to pressure while intracranial inflammation exists, will yield readily to it when that inflammation subsides.

The case is one worthy of note for these reasons, and not less so because of the extended exploration of the brain necessary to discover the abscess in the first instance. To discover pus at a distance of two inches from the surface of the brain, and successfully evacuate it, is an encouragement to future operators. 